\title{
Design and fabrication of two-axis micromachined steel scanners
}

\author{
Y D Gokdel, B Sarioglu, S Mutlu and A D Yalcinkaya \\ Electrical and Electronics Engineering Department, Boğaziçi University, Bebek, TR-34342, Istanbul, \\ Turkey \\ E-mail: arda.yalcinkaya@boun.edu.tr
}

Received 15 November 2008, in final form 18 March 2009

Published 23 June 2009

Online at stacks.iop.org/JMM/19/075001

\begin{abstract}
This paper presents the fabrication and the test results of two-axis micromachined micro-mirror steel scanners developed for display and imaging applications. The novel fabrication method uses the conventional lithography and electrochemical metal etching techniques. A single photomask is used to define the whole structure, resulting in a simple and inexpensive fabrication process. Two different devices are designed, fabricated and characterized to test the proposed methods. Both of them employ the magnetostatic actuation to generate excitation force/torque. First device (Type-A) is a gimballed cantilever one, and it is capable of an optical scanning angle of $11.7^{\circ}$ and $23.2^{\circ}$ in slow- and fast-scan directions, consuming a power of $42 \mathrm{~mW}$ and $30.6 \mathrm{~mW}$, respectively. This structure has a quality factor of 287 in the slow-scan direction and a quality factor of 195 in the fast-scan one. The second device (Type-B) is a gimballed torsional one, and it has an optical scanning angle of $76^{\circ}$ and $5.9^{\circ}$ in slow- and fast-scan directions, consuming $37 \mathrm{~mW}$ and $39 \mathrm{~mW}$, respectively. This structure has a quality factor of 132 in the slow-scan and 530 in the fast-scan directions, respectively. The maximum total optical scanning angles obtained for the slow- and fast-scan axes are $105^{\circ}$ (gimballed torsional device, Type-B) and $42^{\circ}$ (gimballed cantilever device, Type-A).
\end{abstract}

(Some figures in this article are in colour only in the electronic version)

\section{Introduction}

Micro-electro-mechanical mirror scanners (i.e. microscanners) are mainly used in the display/imaging applications where a light beam is scanned in two dimension to create a raster image. There are a number of actuation techniques and mechanical design strategies to implement a micro-scanner aiming to obtain wide optical scan angles for both axes and a high fast-scan frequency to slow-scan frequency ratio. Various materials have been used as the structural element of the scanners. Silicon is by far the most common material [1-4], but there are other alternatives such as metals [5] and polymer-based materials $[6,7]$. There are two common actuation techniques used for micro-scanners: electrostatic and electromagnetic actuation. Electrostatic actuation is widely used for both silicon- [8] and metal-based scanners [9]. Advantages of the electrostatic actuation are zero dc power consumption and the integrated drive mechanism. However, small electrode gaps and relatively high voltages are required for wide scan angles which are not always easily obtained or supplied. Electromagnetic actuation is more flexible, where both soft magnetic materials (such as nickel, steel, permalloy or orthanol) and hard magnetic materials can be utilized [6, 7].

In this paper, we present a novel two-dimensional mirror scanner which uses steel as the structural material. Distinctive to the present device, the scanner is fabricated by using conventional lithography and the electrochemical metal etching technique, making it different from the previously reported steel scanners along with its novel architecture giving better total optical scan angles (TOSA) [5]. Steel is an alternative structural material to traditionally used silicon: it offers a higher Young's modulus $(E)$, a higher torsional modulus $(G)$, a comparable flexural mode frequency coefficient (defined as the ratio between Young' modulus and the material density: $\sqrt{E / \rho}$ ), and a comparable torsion mode frequency coefficient (defined as the ratio between the torsional modulus and the material density: $\sqrt{G / \rho}$ ) [5]. These properties make steel an important candidate for resonant mode actuator applications. Additionally, since steel is a soft magnetic material, electromagnetic actuation can inherently 


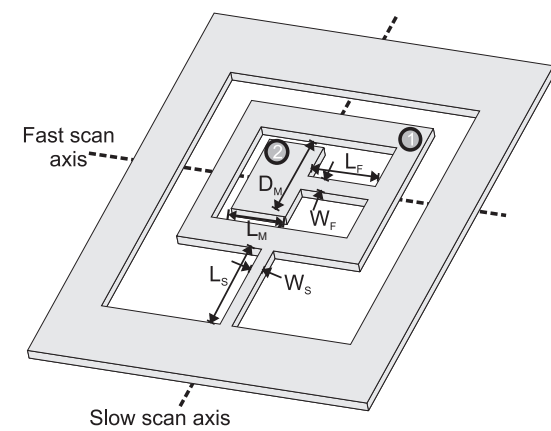

(a)

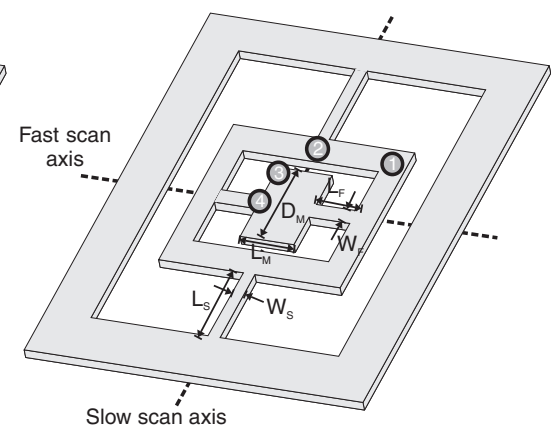

(b)

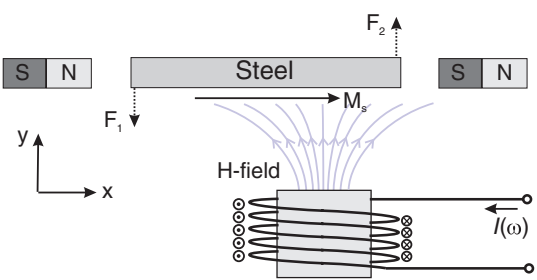

(c)

Figure 1. Schematics and operation of the 2D scanner devices. (a) Gimballed cantilever scanner (Type-A). (b) Gimballed torsional scanner (Type-B). (c) The electromagnetic actuation technique used for both types of the devices.

be utilized to generate excitation force/torque. As a result, similar performances in terms of TOSA, mechanical quality factors and mode frequencies can be obtained by using steel as the structural material.

\section{Device operation}

Schematics of the proposed devices are sketched in figures $1(a)$ and $(b)$, where a slow-scan axis which is orthogonal to the fast-scan axis can be identified. The scanner in figure $1(a)$ is a gimballed cantilever structure (Type-A) in which both cantilever structures generate an out-of-plane bending movement at their resonant frequencies. The device in figure $1(b)$, on the other hand, is a gimballed torsional structure (Type-B) where, both the mirror and the movable frame rotate about the fast and slow axes again at their resonant frequencies. The movable frame is suspended to the anchor through the springs with a length of $L_{S}$ and a width of $W_{S}$, similar to a cantilever design. A mirror, with a size of $D_{M} \times L_{M}$, is gimballed to that movable frame by the suspension with a length of $L_{F}$ and a width of $W_{F}$. The device given in figure 1(a) uses electromagnetic actuation forces to generate out-of-plane displacements. Since the electromagnetic field generating the actuation forces is varying, the most exact calculation would require point-by-point calculation or integration over the magnetic material boundaries. However, considering the small size of the device with respect to the coil size, one can approximate these forces and use two concentrated lumped loads at point-1 (resulting in out-of-plane movement of the movable frame) and at point-2 (out-of-plane movement of the mirror). Thus, the magnitude of the induced force on the movable part can be approximated as [7]

$$
F_{j}(\omega)=M_{s} W t H_{j_{\perp}}(\omega) \quad j=1,2,
$$

where $M_{s}$ is the magnetization vector, $W$ and $t$ are the width and the thickness of the movable section being actuated by the frequency-varying electromagnetic field vector $H_{j_{\perp}}(\omega)$, which is perpendicular to the magnetization vector. The magnetic field, marked as $H$-field in figure $1(c)$, is generated by the electro-coil which is driven by an electrical current. The electro-coil has $7 \mathrm{~mm}$ inner core diameter with 2200 copperwire windings. There are two mechanically fixed permanent magnets, generating approximately $0.8 \mathrm{~T}$ dc field, which yield a predefined magnetization of $M_{S}$ in the steel section, shown as a vector in the $x$-direction in figure $1(c)$. Due to a net force difference, a moment is exerted on the steel scanner. If the rotation axis is in the middle of the lumped point forces of $F_{1}$ and $F_{2}$, the amount of the induced moment is approximately

$$
T(\omega) \simeq\left(F_{1}+F_{2}\right) L_{\mathrm{eff}},
$$

where $L_{\text {eff }}$ stands for the length of the moment arm, calculated as the distance between the lumped force and the center of the rotation axis. There are several advantages associated with this actuation technique such as not having any Joule heating due to the ac current on the scanner, fabrication simplicity and much greater conductor length that can be achieved by using an off-chip coil [7]. Therefore, we choose to use a miniature off-chip coil.

The force or the moment given in equations (1) and (2) displaces the movable parts of the scanner according to Hooke's law. When the frequency of the ac current (thus the ac actuation force/torque) is around the resonance peaks of the vibration modes, the displacements are enhanced by the mechanical quality factors of the respective motion. At resonance, the displacement of the mirror can be calculated as the departure caused by the reacting spring force. Figure 2 shows the finite-element analysis result of the device. According to this analysis, movable frame's fundamental vibration mode (i.e. out-of-plane bending mode) can be used to generate angular displacements about the slow-scan axis for the Type-A scanner, as shown in figure $2(a)$. This 


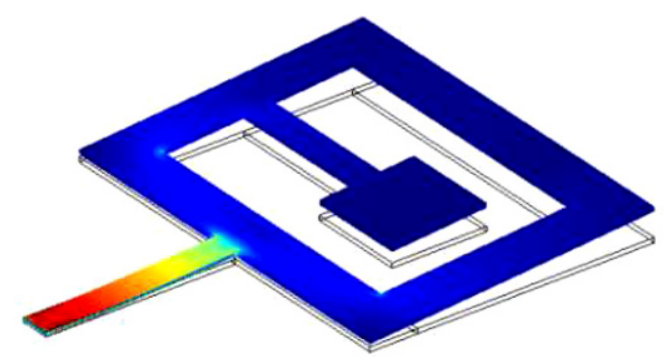

(a)

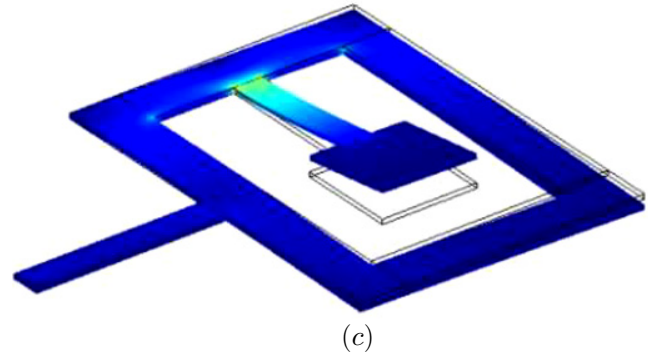

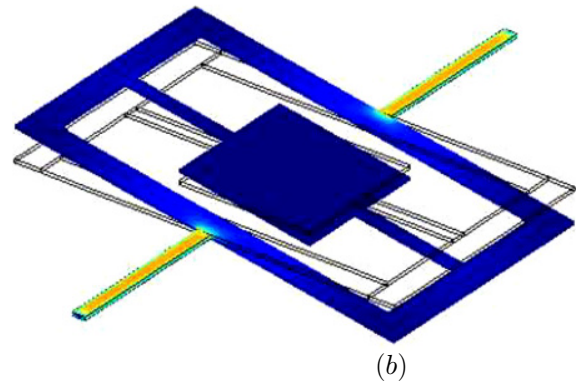

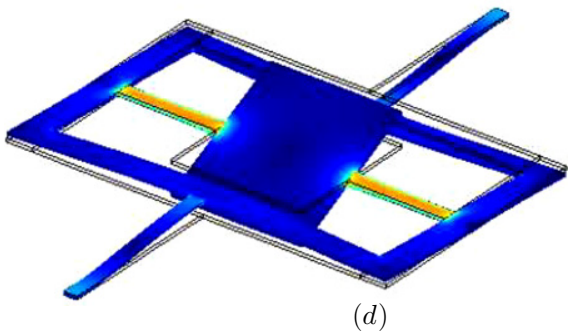

Figure 2. FEA results of the scanning mirrors. (a) Type-A, movable frame bending mode (slow scan). (b) Type-A, mirror bending mode (fast scan). (c) Type-B, movable frame torsion mode (slow scan). (d) Type-B, mirror torsion mode (fast scan).

Table 1. Dimensions (in mm) of the Type-A and Type-B scanners.

\begin{tabular}{llllllll}
\hline Geometry & $W_{S}$ & $L_{S}$ & $W_{F}$ & $L_{F}$ & $D_{M}$ & $L_{M}$ & $t$ \\
\hline Type-A & 1 & 7 & 3 & 1 & 3.5 & 3 & 0.1 \\
Type-B & 0.6 & 5 & 0.8 & 2 & 4 & 4 & 0.1 \\
\hline
\end{tabular}

mode is designed to have a low resonance frequency since it corresponds to the refresh rate of the 2D display [5]. The fast-scan mode of the Type-A scanner has to generate a scan line that is orthogonal to the slow-scan mode, and a suitable candidate is the out-of plane motion of the mirror as depicted in figure $2(b)$. A torsional gimballed scanner (TypeB) device uses conventional torsion modes [3] for both the movable frame and the mirror as shown in figures $2(c)$ and (d), respectively. Each of these two modes will generate scan patterns orthogonal to the other one. The slow and the fastscan modes are excited by supplying ac signal components at the center frequencies of the corresponding resonance peaks. The dimensions of the scanner structures are given in table 1 .

\section{Fabrication}

Application of electrochemical etching to microfabrication dates back to 1955 or even earlier, when it was used to etch germanium and silicon [10]. The cost effectiveness, reduced chemical wastes and effluents, and achievable high precision make electrochemical etching increasingly popular in microsystems [11]. Many alloy compositions, such as stainless steel, can easily be etched by electrochemical etching. Patterning of stainless steel using electrochemical etching can be serial like milling or in batch mode like lithography. Precise electrochemical etching of stainless steel resulting in high-aspect ratio patterns with ultrashort (nano second) voltage pulses using a very sharp tip that is less than a micron away from the etched steel has been shown $[12,13]$. Since this method is a serial process, machining of the work piece takes time, increasing the cost. This is similar to mechanical milling, laser machining and electro discharge machining (EDM) used in industry to machine precise steel parts. A lithographic process is faster and cheaper since it is compatible with batch fabrication. Furthermore, its integration to microsystems is easier and simpler. That is why there have been research efforts to micro-machine steel using a batch mode micro-electro-discharge machining, where lithography and EDM are combined [14]. Similarly, in this work we use a novel fabrication method benefiting from the conventional lithography and electrochemical metal etching. A photoresist mask is patterned on a steel surface using lithography, and then steel is isotropically etched using electrochemical etching. This kind of batch mode electrochemical etching has been studied before [11]. However, it has not been used to fabricate two-axis steel scanners. Steel scanners fabricated so far are not batch fabrication compatible. They are fabricated using mechanical machining [5, 15].

The bulk fabrication method begins with a $100 \mu \mathrm{m}$ thick, stainless steel 301 (SS301) substrate. The substrate is spin coated with a $6 \mu \mathrm{m}$ thick Shipley 1828 photoresist mould which is soft baked at $90{ }^{\circ} \mathrm{C}$ for $60 \mathrm{~s}$. The steel is then exposed to UV light where a nominal exposure time of $6 \mathrm{~min}$ is used with a UV light source that has $1 \mathrm{~mW} \mathrm{~cm}^{-2}$ power. Subsequently, the resist is developed in a developer for $60 \mathrm{~s}$. After the development, the steel is hard baked at $110{ }^{\circ} \mathrm{C}$ for $30 \mathrm{~min}$ in order to make it more durable against the following electrochemical etch process. To perform the electrochemical etch, the steel layer is attached onto an insulator plate holder in order to protect the backside of the material from etching. The steel layer is immersed into an electrochemical cell composed of 1:7 HCl:DI water solution and connected to a current source 
(a)

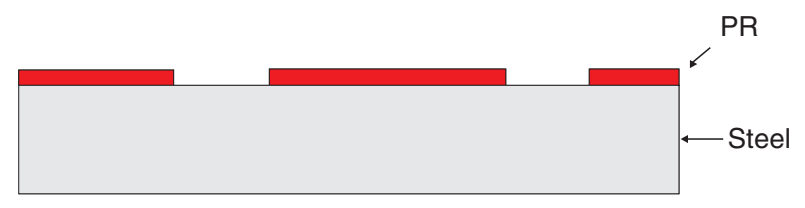

(b)

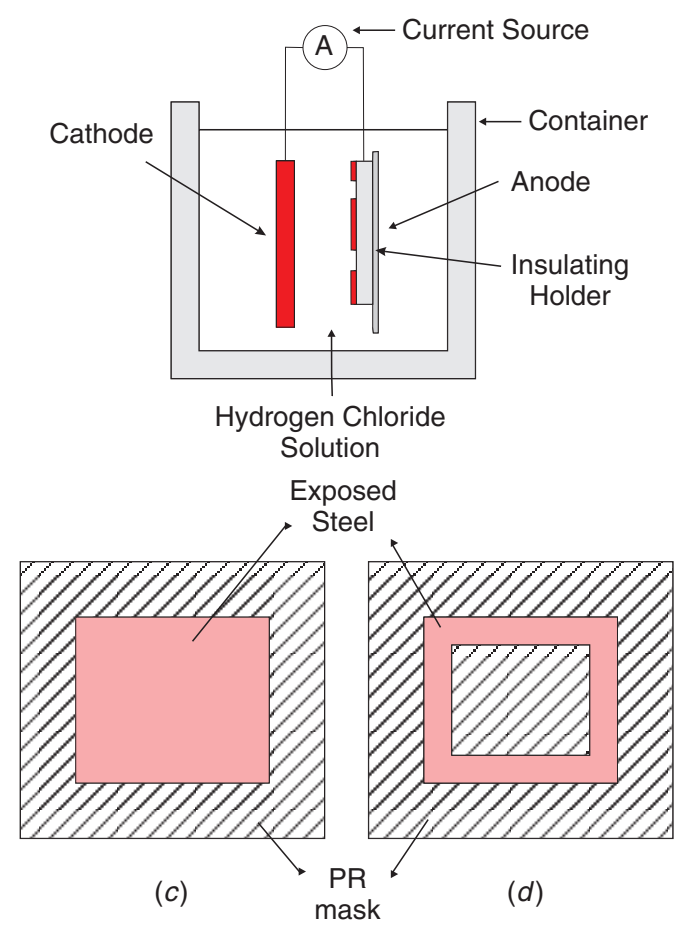

Figure 3. (a) $100 \mu \mathrm{m}$ thick SS301, spin coated and patterned with a $6 \mu \mathrm{m}$ PR mould. (b) Schematics of the electrochemical cell. (c) Standard electrochemical etching mask. (d) Electrochemical etching mask maximizing the current density.

as it is depicted in figure $3(b)$. The parts to be etched form the anode electrode of the electrochemical cell. Conversely, the counter plate, on which ions from the electrolyte get deposited, is the cathode electrode and is also made of steel. They are both connected to a dc power supply which provides current to the cell. Ideally, an anisotropic etching profile can be achieved by employing a slow etching, using a small currentdensity value. However, photoresist is not very durable in electrochemical etch solution and can be delaminated in time. Moreover, because of the poor adhesion of the photoresist on the steel substrate and insufficient post development bake, the disintegration of the photoresist layer along with mask erosions occurs. Consequently, these effects produce some unpredictability in the process, create surface roughness and unintentional etches on the steel surface in various locations that result in poor pattern transfer. Therefore, a long period of hard baking should be preferred; an HMDS adhesion promoter must be utilized and the exposure time of the resist to the $\mathrm{HCl}$ solution has to be diminished to get better etching results. As a result, a very quick and relatively isotopic etching process is preferred instead of a slow and anisotropic one for this ultimate purpose of achieving uniformity on the device. The key of a quick etching process is to increase the current density. Current density can be increased by decreasing the area exposed to the solution or simply by increasing the

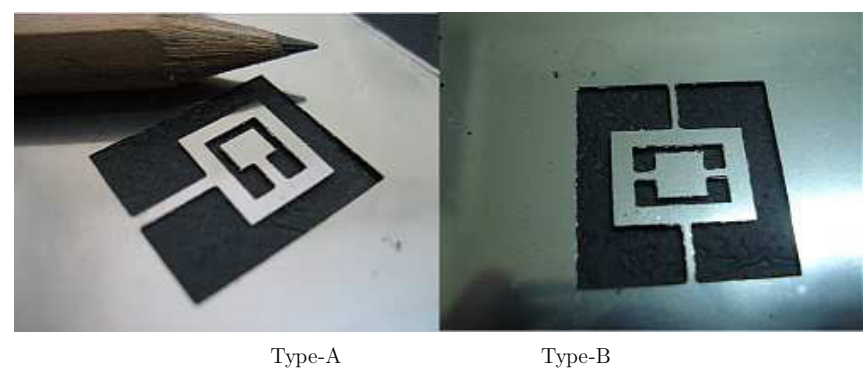

Figure 4. Pictures of the two different designs. Type-A, gimballed cantilever scanner. Type-B, gimballed torsional scanner.

current. Although it is perfectly possible to use an etching mask as shown in figure $3(c)$, eventually the current density would be decreased, making the process longer and destroying the etching uniformity of the device in the end. Moreover, due to the focusing of the electrolytic current distribution at the edges, non-uniform etch profiles in the form of W shape can occur in the patterns with wide openings [16, 17]. For a given maximum de current (3.3 A in this case), and a given etch pattern, one solution would be to decrease the exposed area as depicted in figure $3(d)$, which renders the same pattern as in figure $3(c)$. The width of the exposed area in this mask is $100 \mu \mathrm{m}$ but for better uniformity, smaller dimensions can be used with ease. It is important to note that this situation is only true for etch-through processes. This masking scheme enables the etching of $100 \mu \mathrm{m}$ thick stainless steel plates with a current density of approximately $9 \mathrm{~A} \mathrm{~cm}^{-2}$. This creates a one-to-one isotropy which means that approximately $100 \mu \mathrm{m}$ undercut occurs for $100 \mu \mathrm{m}$ depth. However, the undercut can be compensated with no trouble by simply drawing the masks accordingly. The devices are etched in just, 4-5 min with a rate of approximately $25 \mu \mathrm{m} \mathrm{min}^{-1}$ depending on the structure. Finally, after electrochemical etch, the PR mould is stripped off. Produced devices are shown in figure 4. Two nickel-plated magnets which have the dimensions of $1 \mathrm{~mm} \times$ $5 \mathrm{~mm} \times 1.5 \mathrm{~mm}$ and weight of $55 \mathrm{mg}$ are attached onto the anchor frames of the devices in order to be able to define the magnetization on the movable parts. An off-the-shelf coil is optimally placed near the steel mirror for actuation.

\section{Test and characterization results}

Scanner frequency response is characterized using a setup that consists of a laser Doppler vibrometer (LDV), a function generator and an oscilloscope. Communication between the oscilloscope and the function generator is accomplished by the GPIB interface. The LDV sends a laser beam to the section of interest and measures the Doppler shift of the returning beam to give a voltage at its output which carries the signature of the vibration velocity of that particular section. In the experiments, both the coil excitation voltage (current) and the LDV output voltage are sinusoidal signals. For a given setting of the LDV, output voltage is converted to velocity by scaling it with a constant. Figure 5 shows the mechanical transfer characteristics of the Type-A device as a function of the operation frequency. This plot is obtained by driving the 
(a)

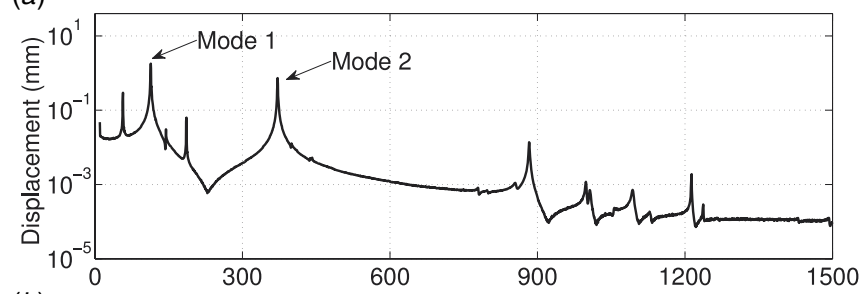

(b)

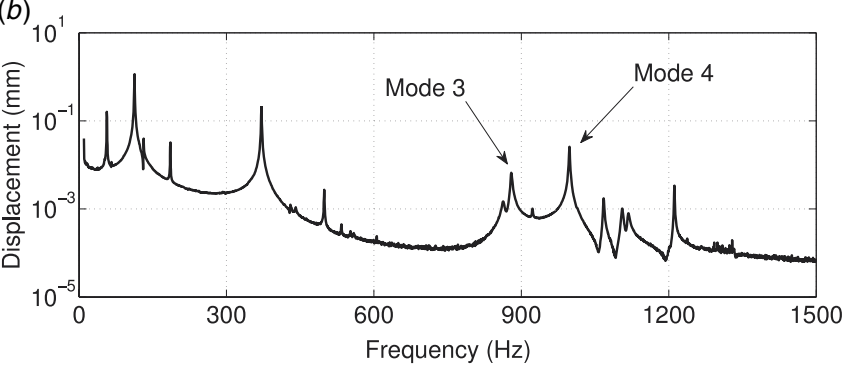

Figure 5. Vibration displacement of $(a)$ the movable frame (point-1). (b) The mirror (point-2) of the Type-A scanner as a function of frequency.

electro-coil with a constant magnitude current, varying the frequency and collecting the vibration displacement by the LDV (Polytec OFV 2500). As can be seen from this figure, there are two points of interest on the device, namely point-1 and point-2 (whereas the characterization of the torsionaltorsional structure is made considering four different points in order to increase the accuracy of the results; refer to figures $1(a)$ and $(b)$ for the locations of these points), which indicate the slow-scan and the fast-scan operations, respectively. Mode-1 corresponds to the out-of-plane displacement of the movable frame at point-1, generating a scan line at $112 \mathrm{~Hz}$. This resonance peak, given in figure 6(a) in detail, results in a quality factor of 287 in air. Mode- 1 is chosen as the slow-scan mode for two-dimensional scanning. Mode- 2 corresponds to the torsional movement of the movable frame which has a resonance frequency of $371 \mathrm{~Hz}$. Subsequently, point-2 is used in the following measurements to define the characteristics of the fast-scan operation. The mirror produces the maximum scan line by making an outof-plane displacement at $882 \mathrm{~Hz}$ which is defined as mode-3 in figure $5(b)$. This resonance peak generates a scan line that is orthogonal to the scan line produced by mode- 1 , thus can be chosen as the fast-scan operation mode. The mechanical quality factor for this mode is measured to be approximately 200 in air. Mode-4 in figure 5(b), depicts the torsional movement of the mirror. For the Type-A scanner, fast-scan frequency (mode-3) to slow-scan frequency (mode-1) ratio is 7.87 for resonant actuation of both the modes. If nonresonant actuation is used for the slow scan, e.g. $60 \mathrm{~Hz}$ refresh rate, the ratio between the fast-scan frequency and the slowscan frequency becomes 14.7, which implies that one can write approximately 15 lines in the vertical direction using the present device as a $2 \mathrm{D}$ display.

Figure 7 shows the laser scanning experiments of the Type-A scanner. The laser beam is positioned on the mirror, and the device is actuated in slow- and fast-scan resonances, respectively. A white, scaled metric screen is placed as a screen
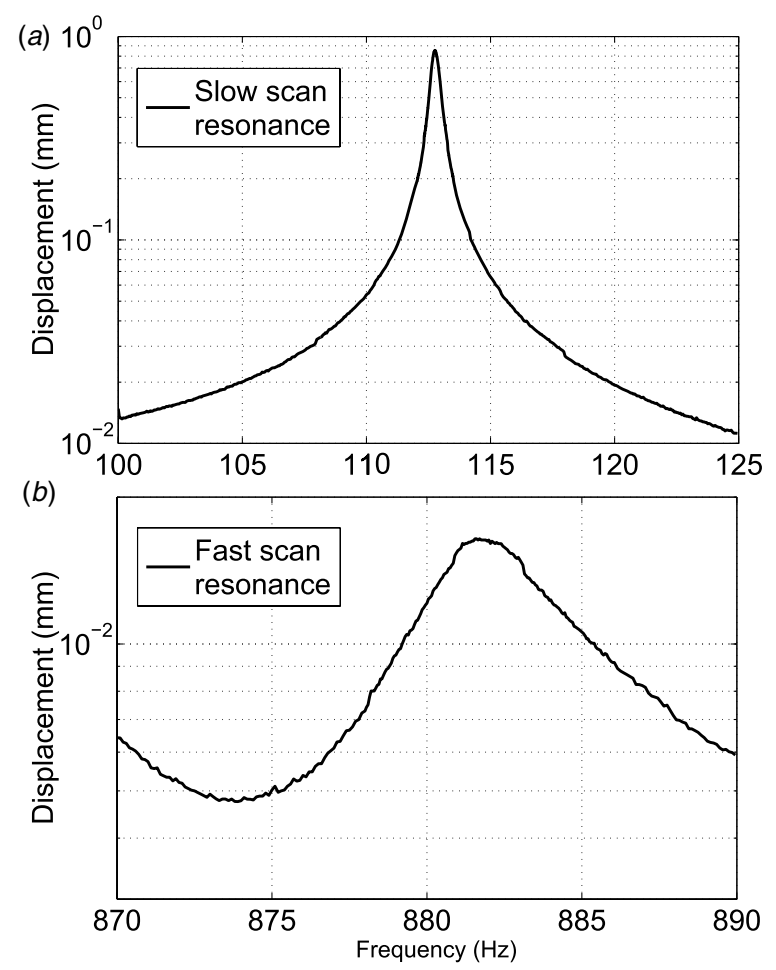

Figure 6. (a) Out-of-plane displacement resonant peak of the movable frame. (b) Out-of-plane displacement resonant peak of the mirror for the Type-A device.

for the 2D scanner, where reflected laser beam generates a onedimensional scan line for each axis. As can be seen from figure 7(a), the slow-scan motion produces a scan line corresponding to a TOSA of $11.7^{\circ}$ at a drive power of $42 \mathrm{~mW}$. Similarly, the fast-scan operation at a drive level of $30.6 \mathrm{~mW}$ results in a TOSA of $23.2^{\circ}$, as shown in figure $7(b)$. Here, it is important to note that with large optical scan angles, steel mirror dynamic deformation, which produces slightly curved edges on the scan lines, is more apparent as shown in figures 7(a) and $(c)$. In order to characterize two-dimensional scanning capability of the scanner, slow- and fast-scan drive signals are superimposed resulting in a Lissajous pattern shown in figure 7(c). Similar experiments are repeated for the Type-B device. As a result, slow-scan motion of the TypeB scanner has a TOSA of $76^{\circ}$ at a drive power of $37 \mathrm{~mW}$, whereas the fast-scan motion has a TOSA of $5.9^{\circ}$ at a drive power of $39 \mathrm{~mW}$ as is shown in figure 8 .

Actuation power linearity is studied for both types of scanners. The experiment is performed by cranking up the drive power of the coil and measuring the scan line (thus the optical scan angle) at each data point at the resonance peak of the mode. The result of this experiment is given in figure 8 , where TOSA of the slow- and fast-scan modes are plotted versus the power of the driving alternative signal of the electro-coil. As can be seen from figure 8(a), for the Type-B scanner, since the mirror displacement is linearly proportional to the magnetic flux (and therefore the coil current), there is a dependence resembling a square-root relationship between the scan angle and the drive power. Since the mechanical quality factor of the device varies with the actuation power, there is no exact square-root behavior. Figure $8(b)$ shows the 


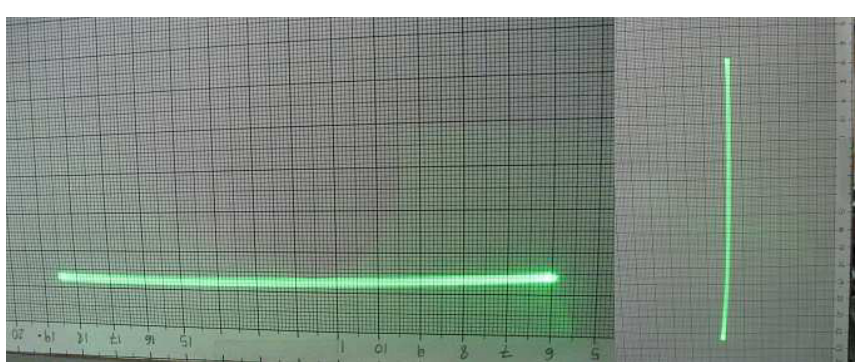

(a)

(b)

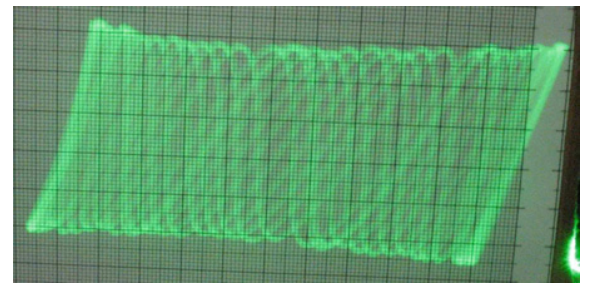

(c)

Figure 7. Scan line characterization results for the Type-A device. (a) Horizontal total optical scan line is $11.7^{\circ}$ at a drive power of $42 \mathrm{~mW}$. (b) Vertical total optical scan angle (TOSA) is $23.2^{\circ}$ at a drive power of $30.6 \mathrm{~mW}$. (c) Lissajous pattern.

characteristic for the fast axis of the Type-B scanner, where the drive power is changed in a smaller range, resulting in a similar behavior like the slow axis. As can be seen in figure 8, a maximum slow axis TOSA of $105^{\circ}$ is possible with the Type-B device. It is apparent from these experiments that the drive power is linearly proportional to the square of the TOSA.

\section{Control setup}

\subsection{Actuation}

Actuation of the scanner and modulation of the laser source are done via a control circuit whose schematic is shown in figure 9. The circuit is composed of a Spartan 3AN field-programmable gate array (FPGA) development board and additional active and passive components in order to convert data and drive the coil and laser diode. The FPGA board acts as the main control unit and generates both the modulation and actuation signals as digital words. The digital actuation signal, generated in the FPGA, is then converted to an analog signal via a digital-toanalog converter (DAC) which drives the electro-coil.

The actuation signal is basically a combination of two individual signals, namely slow-scan mode and fast-scan mode actuation signals, which are superimposed by the FPGA set. Both the mode signals and the resulting superimposition have 8-bit depth. The frequency data that are employed for the generation of the signals are stored in the ROM of the development board and it can be adjusted by knobs and buttons of the board, in real time. The fast-scan mode is driven to resonance, while off-resonance driving is applied for the slowscan mode operation. The slow-scan mode signal comprises a simple ramp signal at $100 \mathrm{~Hz}$. The fast-scan modulation signal is a pure sinusoidal signal at $1.2 \mathrm{kHz}$ and it is created by using sine values stored in a look up table (LUT). Synchronizing the actuation signals appropriately and keeping a constant phase between them are crucial, since they define the position of the rows and columns of the display along with the contrast ratio of the image to be created. Two different methods can be pursued to accomplish this: (1) to make one of the actuation signals an integer multiple of the other (off-resonance actuation) [18] and (2) to use a synchronous pulse obtained from a feedback loop of one signal in order to start the other one (resonant actuation) [5]. The first method may need the amplification of the off-resonance signal. On the other hand, for the latter one, starting one signal abruptly may create harmonics which actuate unwanted modes of the scanner and the result of this can be a distorted image. In this work, the second method is used. The synchronization signal which starts the first fastscan operation is created when the slow-scan mode operation is finished. The distortions are minimized by adjusting the slow-scan frequency accordingly.

\subsection{Modulation}

The modulation of the light source is performed by a buffer, which drives the laser using an input signal from the FPGA

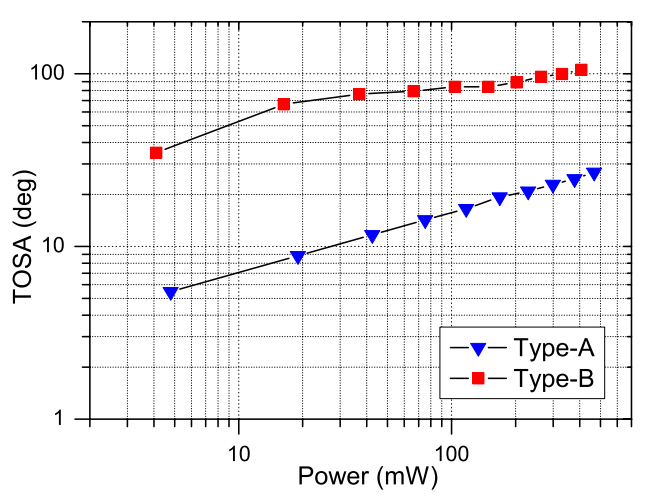

(a)

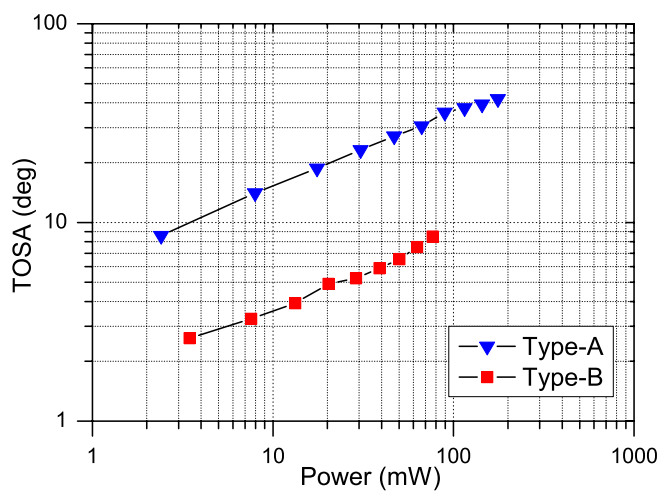

(b)

Figure 8. Drive power versus TOSA relationship for the cantilever-type gimballed (Type-A) scanner and the torsional-type gimballed (Type-B) scanner. (a) Slow-scan characteristics. (b) Fast-scan characteristics. 


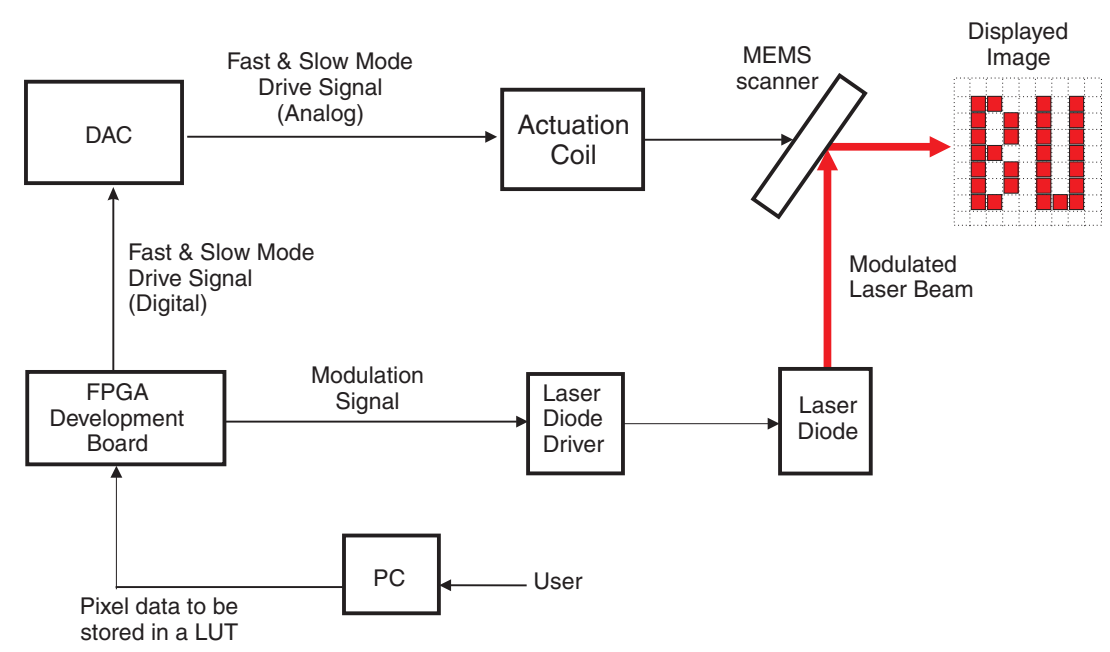

Figure 9. Test setup used for modulation of the light source and the drive control of the electro-coil.
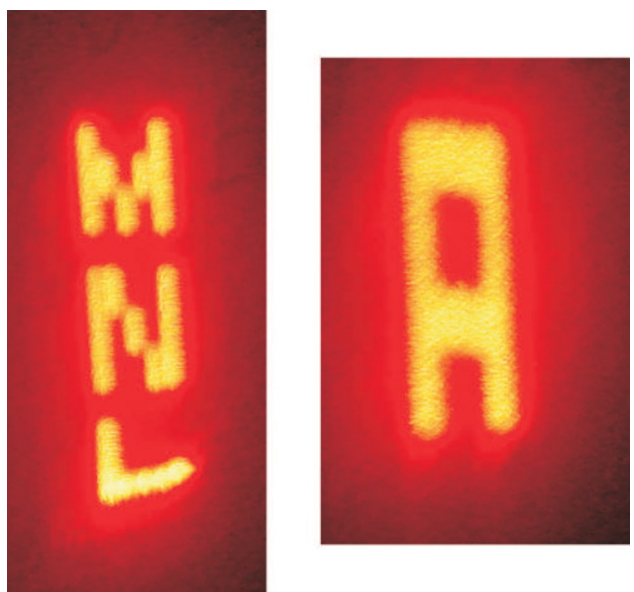

Figure 10. Two examples of the displayed character patterns: the left one is ' $\mathrm{MNL}$ ' text (size: $4 \mathrm{~mm} \times 12 \mathrm{~mm}$ ) and the right one is the 'A' character.

board. The modulation signal is created from the information related to the fast-scan and the slow-scan actuation signals. The beginning of the fast-scan signal corresponds to the creation of the first pixel for that particular row. Moreover, the value of the row counter located in the FPGA kit is increased at the beginning of each fast-scan period until the end of the slow-scan period. The nonlinear portions of the fast-scan mode sinusoidal signal are not used in order to obtain a non-distorted image. The number of pixel for one row is virtually limited by the laser spot size. The pixel clock frequency, which plays an important role in achievable resolution, is dependent on the physical characteristics of the laser diode, the performance of the modulation circuitry and the target contrast level [19].

Both unilateral and bilateral scanning are possible for the MEMS scanners and they are investigated in detail in [5]. The scanner velocity is highest at the center of the mirror, and therefore the pixels at the center are larger. Some form of velocity compensation should be used to achieve the best image quality. The characters shown in figure 10 are formed by applying unilateral scanning with no velocity compensation. The mirror under test is shown in figure 11. A hard-plastic holder, produced from a Plexiglass substrate by employing

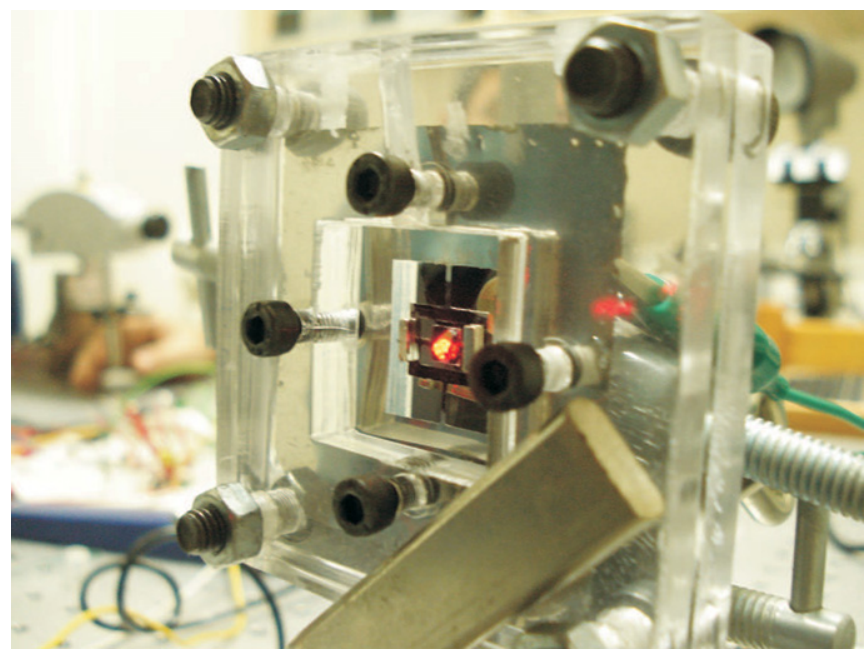

Figure 11. Photo of the experimental control setup.

a laser cutter, is implemented to prevent the energy loss mechanism through the anchor points.

\section{Conclusion}

In this paper, a new fabrication alternative is presented for steel micromachining. The fabrication method uses only one photomask and a single lithography step followed by an etch step. Reasonable etch uniformity with an etch rate of $25 \mu \mathrm{m} \mathrm{min}^{-1}$ is obtained. A family of scanners is fabricated, and two of these scanners, afore-mentioned gimballed cantilever (Type-A) and gimballed torsional (TypeB) devices, are presented and shown that they are capable of being used in the display applications. Present devices in this work are the first two-dimensional steel micro mirrors. The Type-A device gives a maximum TOSA of $11.7^{\circ}$ in the slow-scan axis and $23.2^{\circ}$ in the fast-scan axis in its resonance modes $(112 \mathrm{~Hz}$ for slow-scan movement and $882 \mathrm{~Hz}$ for fast-scan movement) at a drive power of $42 \mathrm{~mW}$ and $30.6 \mathrm{~mW}$, respectively. On the other hand, the Type-B device in resonance, generates a fast-scan TOSA of $5.9^{\circ}$ and a 
slow-scan TOSA of $76^{\circ}$ for drive powers of $39 \mathrm{~mW}$ and $37 \mathrm{~mW}$, respectively. The scanner devices are operated in ambient air and resulting mechanical quality factors for the scan modes are sufficient $(Q>100)$ to have low power consumption. For the Type-A scanner, the fast-scan frequency to slow-scan frequency ratio is 7.87 for resonant actuation of both modes. If non-resonant actuation is used for the slow scan, e.g. $60 \mathrm{~Hz}$ refresh rate, the ratio between fast- and slow-scan frequencies becomes 14.7, which implies that one can write approximately 15 lines in the vertical direction using the present device as a 2D display. Experiments showed that the drive power is approximately proportional to the square of the TOSA. A simple two-dimensional display system is constructed by using the fabricated steel scanner and accompanying driving and signal processing circuitry. Obtained images show the potential of the steel scanners in imaging applications.

\section{Acknowledgments}

This project is financed by the Turkish National Science Agency (TUBITAK, project number 107E053) and by the Scientific Research Projects Office Bogazici University (BAP 07A202).

\section{References}

[1] Ji C-H, Choi M, Kim S-C, Song K-C, Bu J-U and Nam H-J 2007 Electromagnetic two-dimensional scanner using radial magnetic field IEEE J. Microelectromech. Syst. 16 989-96

[2] Yang H-A, Tang T-L, Lee S T and Fang W 2007 A novel coilless scanning mirror using eddy current Lorentz force and magnetostatic force IEEE J. Microelectromech. Syst. 16 511-20

[3] Yalcinkaya A D, Urey H, Montague T, Brown D and Sprague R 2006 Two-axis electromagnetic microscanner for high reslolution displays IEEE J. Microelectromech. Syst. 15 786-94

[4] Jain A, Kopa A, Yingtian P, Fedder G K and Huikai X 2004 A two-axis electrothermal micromirror for endoscopic optical coherence tomography IEEE J. Sel. Top. Quantum Electron. 10 636-42

[5] Urey H 2002 Torsional MEMS scanner design for high-resolution display systems Optical Scanning II, Proc. SPIE (Seattle, USA) 4773 27-37
[6] Yalcinkaya A D, Ergeneman O and Urey H 2007 Polymer magnetic scanner for bardcode reader applications Sensors Actuators A 135 236-43

[7] Isikman S O, Ergeneman O, Yalcinkaya A D and Urey H 2007 Modeling and characterization of soft magnetic film actuated 2d scanners IEEE J Sel. Top. Quantum Electron. 13 283-9

[8] Schenk H, Durr P, Haase T, Kunze D, Sobe U, Lakner H and Kuck H 2000 Large deflection micromechanical scanning mirrors for linear scans and pattern generation IEEE J Sel. Top. Quantum Electron. 6 715-22

[9] Buhler J, Funk J, Korvink J G and Steiner F P 1997 Electrostatic aluminum micromirrors using double pass metallization IEEE J. Microelectromech. Syst. 6 128-34

[10] Uhlir A 1956 Electrolytic shaping of germanium and silicon Bell Syst. Tech. J. 35 333-47

[11] Datta M and Landolt D 2000 Fundamental aspects and applications of electrochemical microfabrication Electrochim. Acta $\mathbf{4 5}$ 2535-58

[12] Kirchner V, Cagnon L, Schuster R and Ertl G 2001 Electrochemical machining of stainless steel microelements with ultrashort voltage pulses Appl. Phys. Lett. 79 1721-3

[13] Kim B H, Ryu S H, Choi D K and Chu C N 2005 Micro electrochemical milling J. Micromech. Microeng. 15 124-9

[14] Richardson M T and Gianchandani Y B 2008 Achieving precision in high density batch mode micro-electro-discharge machining J. Micromech. Microeng. 18015002

[15] Akedo J, Park J-H and Sato H 2007 High-speed metal-based optical microscanners using stainless-steel substrate and piezoelectric thick films prepared by aerosol deposition method Sensors Actuators A 135 86-91

[16] West A C, Madore C, Matlos M and Landolt D 1992 Shape changes during through-mask electrochemical micromachining of thin metal films J. Electrochem. Soc. 139-2 499-506

[17] Mineta T 2004 Electrochemical etching of a shape memory alloy using new electrolyte solutions J. Micromech. Microeng. 14 76-80

[18] Wang W-C and Reinhall P G 2008 Scanning polymeric waveguide design of a 2-D display system IEEE J. Disp. Technol. 4 28-38

[19] Wine D W, Helsel M P, Jenkins L, Urey H and Osborn T D 2000 Performance of a biaxial MEMS-based scanner for microdisplay applications Proc. SPIE Conf. on MOEMS and Miniaturized Systems (Santa Clara, CA) 4178 186-96 